\title{
Primes in almost all short intervals
}

by

\section{Alessandro ZaCCAGnini (Parma)}

1. Introduction. The object of this paper is to extend the range of validity of a well-known result of prime number theory. We deal with the Selberg integral

$$
J(x, h):=\int_{x}^{2 x}\left|\pi(t)-\pi(t-h)-\frac{h}{\log t}\right|^{2} d t .
$$

The Prime Number Theorem suggests that $J(x, h)$ should be of lower order of magnitude than $x h^{2}(\log x)^{-2}$, at least when $h$ is not too small with respect to $x$, and the Brun-Titchmarsh inequality trivially implies $J(x, h) \ll$ $x h^{2}(\log x)^{-2}$ provided only that $h \geq x^{\varepsilon}$ for some fixed $\varepsilon>0$.

We prove the following

Theorem. We have

$$
J(x, h) \ll \frac{x h^{2}}{(\log x)^{2}}\left(\varepsilon(x)+\frac{\log \log x}{\log x}\right)^{2}
$$

provided that $x^{1 / 6-\varepsilon(x)} \leq h \leq x$, where $0 \leq \varepsilon(x) \leq 1 / 6$ and $\varepsilon(x) \rightarrow 0$ as $x \rightarrow \infty$.

It is well known that Huxley's density estimates [5] for the zeros of the Riemann zeta-function yield $J(x, h)=o\left(x h^{2}(\log x)^{-2}\right)$, but only for $h \geq x^{1 / 6}(\log x)^{C}$, for some $C>0$. The weaker result with $h \geq x^{1 / 6+\varepsilon}$ is proved in Saffari and Vaughan [8], Lemma 5, and in [13], where an identity of Heath-Brown (Lemma 1 of [3]) is used.

This paper is inspired by Heath-Brown's extension [4] of Huxley's Theorem [5] that

$$
\pi(x)-\pi(x-h) \sim h(\log x)^{-1}
$$

to the range $h \geq x^{7 / 12-\varepsilon(x)}$. This was achieved by means of another identity (see (2.2) of [4], or Lemma 2 below), thereby avoiding a direct appeal to the

1991 Mathematics Subject Classification: Primary 11N05. 
properties of the zeros of the Riemann zeta-function, besides Vinogradov's zero-free region. We extend this approach to the above integral.

An immediate consequence of this result is that if $x^{1 / 6-\varepsilon(x)} \leq h \leq x$ then for "almost all" $n \in[x, 2 x] \cap \mathbb{N}$ we have $\pi(n)-\pi(n-h) \sim h(\log n)^{-1}$. Here "almost all" means that the above asymptotic equality fails for at most $o(x)$ values of $n \in[x, 2 x] \cap \mathbb{N}$. Relaxing our demand to $\pi(n)-\pi(n-h) \gg$ $h(\log n)^{-1}$ for almost all $n$ 's, one can take $h$ even smaller, and the best result up to date is due to Jia [6] who showed that $h \geq x^{1 / 20+\varepsilon}$ is acceptable, provided that $x$ is large enough.

I thank Alberto Perelli for his unfailing help and János Pintz for some helpful suggestions. Many thanks are due to the referee for a very careful reading of my manuscript and numerous useful remarks.

2. Preliminaries. We assume throughout that $x$ is sufficiently large. For the sake of brevity we set $\mathcal{L}:=\log x$. Our estimates will be uniform with respect to all parameters but $k_{0}$, which will eventually be chosen as 4. For ease of reference, our notation is consistent, as far as possible, with the notation in [4], and will be introduced at appropriate places. A few comments on the proof are collected at the end of the paper.

Lemma 1. The Theorem follows from the estimate

$$
J^{\prime}(x, \theta):=\int_{x}^{2 x}\left|\pi(t)-\pi(t-\theta t)-\frac{\theta t}{\log t}\right|^{2} d t \ll \frac{x^{3} \theta^{2}}{\mathcal{L}^{2}}\left(\varepsilon(x)+\frac{\log \log x}{\log x}\right)^{2},
$$

uniformly for $x^{-5 / 6-\varepsilon(x)} \leq \theta \leq 1$.

Lemma 2 (Linnik-Heath-Brown's identity). For $z>1$ we have

$$
\log (\zeta(s) \Pi(s))=\sum_{k \geq 1} \frac{(-1)^{k-1}}{k}(\zeta(s) \Pi(s)-1)^{k}=\sum_{k \geq 1} \sum_{p \geq z} \frac{1}{k p^{k s}},
$$

where

$$
\Pi(s):=\prod_{p<z}\left(1-\frac{1}{p^{s}}\right) .
$$

For Lemma 1 see the proof of Lemma 6 of [8]. Lemma 2 follows from (2.2) $-(2.3)$ of $[4]$.

For $t \in[x, 2 x]$ we use the interval $\mathcal{I}=\mathcal{I}(t, \theta)=(t-\theta t, t]$, and a parameter $z$ satisfying

$$
x^{1 / k_{0}}<z \leq x^{1 / 3} .
$$

We pick out the coefficients in the above identity for the terms with $n \in \mathcal{I}$. 
We have

$$
\sum_{k \geq 1} \frac{1}{k}\left|\left\{p: p^{k} \in \mathcal{I}, p \geq z\right\}\right|=\pi(t)-\pi(t-\theta t)+O\left(\theta x^{1 / 2}+\log x\right),
$$

the contribution from prime powers being negligible. Now the Dirichlet series for $\zeta(s) \Pi(s)-1$ is $\sum_{n \geq z} a(n) n^{-s}$ where $a(1)=0$ and $a(n)=0$ unless all prime factors of $n$ are $\geq z$, in which case $a(n)=1$. Furthermore, the Dirichlet series for $(\zeta(s) \Pi(s)-1)^{k}$ is $\sum_{n \geq z} a_{k}(n) n^{-s}, a_{k}$ being the $k$-fold Dirichlet convolution of $a$ with itself. This means that $a_{k}(n)=0$ unless $n \geq z^{k}$ and $p \geq z$ for all $p \mid n$. Hence there are no terms $n^{-s}$ with $n \in \mathcal{I}$ and $k \geq k_{0}$, and we may consider only the values $k<k_{0}$.

As pointed out in Section 2 of [4], the above identity does not give suitable Dirichlet polynomials at once, and we first need to approximate the above Dirichlet series by manageable Dirichlet polynomials. We set

$$
\zeta_{t}(s):=\sum_{n \leq t} \frac{1}{n^{s}}
$$

We introduce parameters $z_{1} \in[3, z)$ and $z_{2}:=z_{1}^{\delta}$, where $\delta \geq 2$ and define $v_{n}$ by means of

$$
\Pi_{0}(s):=\prod_{p<z_{1}}\left(1-\frac{1}{p^{s}}\right)=\sum_{n \geq 1} \frac{\mu(n) v_{n}}{n^{s}} .
$$

Then define $\Pi_{1}(s):=\Pi(s) \Pi_{0}(s)^{-1}, L$ to be the integer such that $z_{1}^{L} \leq$ $2 x<z_{1}^{L+1}$ and

$$
\Pi_{2}(s):=\sum_{n<z_{2}} \frac{\mu(n) v_{n}}{n^{s}}, \quad \Sigma_{m}(s):=\sum_{z_{1} \leq p<z} \frac{1}{p^{m s}}
$$

for $m=1, \ldots, L$. Finally, we set

$$
\Pi^{*}(s):=\prod_{m=1}^{L} \Pi_{m}^{*}(s) \quad \text { where } \quad \Pi_{m}^{*}(s):=\sum_{l=0}^{L / m} \frac{(-1)^{l}}{l ! m^{l}} \Sigma_{m}(s)^{l} .
$$

We remark that our choice of the parameters ensures that the coefficient of $n^{-s}$ in $\Pi_{1}(s)$ is the same as the coefficient of $n^{-s}$ in $\Pi^{*}(s)$. We now introduce the Dirichlet polynomials we shall work with. Let $B, C$, and $D$ be integers such that

$$
t / 2<2^{B} \leq t, \quad z_{2} / 2<2^{C} \leq z_{2}, \quad z / 2 \leq 2^{D}<z,
$$

and set

$$
\zeta_{t}(s)=\sum_{b=0}^{B} X_{b}(s), \quad X_{b}(s):=\sum_{2^{-1-b} t<n \leq 2^{-b} t} n^{-s},
$$




$$
\begin{aligned}
& \Pi_{2}(s)=\sum_{c=0}^{C} Y_{c}(s), \quad Y_{c}(s):=\sum_{2^{-1-c} z_{2}<n \leq 2^{-c} z_{2}} \mu(n) v_{n} n^{-s}, \\
& \Sigma_{m}(s)=\sum_{d=0}^{D} Z_{d}^{(m)}(s), \quad Z_{d}^{(m)}(s):=\sum_{\substack{2^{-1-d} z<p \leq 2^{-d} z \\
p \geq z_{1}}} p^{-m s} .
\end{aligned}
$$

Hence, for suitable coefficients $c_{m, h}$, we have

$$
\left(\zeta_{t}(s) \Pi_{2}(s) \Pi^{*}(s)\right)^{h}=\sum_{m=1}^{M(h)} c_{m, h} W(s ; m, h),
$$

where the Dirichlet polynomials $W$ have the form

$$
W(s ; m, h)=W_{X}(s ; m, h) W_{Y}(s ; m, h) W_{Z}(s ; m, h),
$$

with

$$
\begin{aligned}
W_{X}(s) & :=\prod_{i=1}^{h} X_{b_{i}}(s), \quad W_{Y}(s):=\prod_{i=1}^{h} Y_{c_{i}}(s), \\
W_{Z}(s) & :=\prod_{m=1}^{L} \prod_{i=1}^{I_{m}} Z_{d_{i}}^{(m)}(s),
\end{aligned}
$$

where each $I_{m}$ is $\leq h L / m$, and we dropped $m$ and $h$ for brevity. Writing

$$
X_{i}:=2^{-1-b_{i}} t, \quad Y_{i}:=2^{-1-c_{i}} z_{2}, \quad Z_{i}:=2^{-1-d_{i}} z,
$$

and $I=\sum_{m} I_{m}$, we have

$$
W(s ; m, h)=\sum_{N_{1}<n \leq N_{2}} \frac{e_{m, h}(n)}{n^{s}},
$$

where

$$
N_{1}:=\prod_{i=1}^{h} X_{i} Y_{i} \cdot \prod_{m=1}^{L} \prod_{i=1}^{I_{m}} Z_{i} \quad \text { and } \quad N_{2}:=2^{2 h+I} N_{1} .
$$

Since we are interested in the coefficients of the terms $n^{-s}$ with $n \in \mathcal{I}(t, \theta)$, we may obviously discard those sums $W(s)$ with $N_{1} \geq t$ or $N_{2} \leq t / 2$, leaving, after relabeling,

$$
\sum_{m=1}^{N(h)} c_{m, h} W(s ; m, h)
$$

say. As usual, we denote by $d_{m}(n)$ the coefficient of $n^{-s}$ in $\zeta^{m}(s)$. We now state the following results, the first being a consequence of Theorem 2 of Shiu [9]. 
Lemma 3. For fixed $\varepsilon>0$ and $m, h \in \mathbb{N}$ we have

$$
\sum_{x \leq n \leq x+y} d_{m}^{h}(n) \ll_{\varepsilon, m, h} y(\log x)^{m^{h}-1}
$$

uniformly for $x^{\varepsilon} \leq y \leq x$.

Lemma 4. For $t \in[x, 2 x]$ there exist Dirichlet polynomials $W(s ; m, h)$ satisfying (2.3)-(2.11) such that

$\sum_{n \in \mathcal{I}(t, \theta)} a_{k}(n)=\sum_{h=0}^{k}(-1)^{k-h}\left(\begin{array}{l}k \\ h\end{array}\right) \sum_{m=1}^{N(h)} c_{m, h} \sum_{n \in \mathcal{I}(t, \theta)} e_{m, h}(n)+O\left(x \theta \mathcal{L}^{3 k} \delta^{-\delta / 3}\right)$ when $z_{1} z_{2} \leq x^{1 / 8}$ and $\delta \geq\left(\log \log z_{1}\right)^{2}$.

The proof is quite similar to the proof of Lemma 3 of [4], using Lemma 3 above. We omit it for brevity. Set

$$
\Sigma(h, t, \theta):=\sum_{m=1}^{N(h)} c_{m, h} \sum_{n \in \mathcal{I}(t, \theta)} e_{m, h}(n)
$$

(here a minor clash with the notation of [4] occurs). Then

$$
S(t, \theta):=\pi(t)-\pi(t-\theta t)=\sum_{1 \leq k<k_{0}} \sum_{h=0}^{k} \alpha(h, k) \Sigma(h, t, \theta)+O(E(t, \theta, \delta)),
$$

say, where $\alpha(h, k) \ll 1$ and $E(t, \theta, \delta) \ll \theta\left(x^{1 / 2}+x \mathcal{L}^{3 k} \delta^{-\delta / 3}\right)$ by $(2.1),(2.2)$ and Lemma 4 . Our aim is to prove that each $\Sigma$ can be written as

$$
\Sigma(h, t, \theta)=\theta \mathfrak{M}(h, t)+\mathfrak{R}(h, t, \theta),
$$

where $\mathfrak{M}(h, t)$ is independent of $\theta$ and $\mathfrak{R}(h, t, \theta)$ is small in $L^{2}$ norm over $[x, 2 x]$. In fact, assume that (2.12) holds for suitable $\mathfrak{M}$ and $\mathfrak{R}$, and let

$$
\begin{aligned}
& \mathfrak{M}(t):=\sum_{1 \leq k<k_{0}} \sum_{h=0}^{k} \alpha(h, k) \mathfrak{M}(h, t), \\
& \mathfrak{R}(t, \theta):=\sum_{1 \leq k<k_{0}} \sum_{h=0}^{k} \alpha(h, k) \mathfrak{R}(h, t, \theta),
\end{aligned}
$$

so that $S(t, \theta)=\theta \mathfrak{M}(t)+\mathfrak{R}(t, \theta)+O(E(t, \theta, \delta))$. Since $(a+b+c)^{2} \ll$ $a^{2}+b^{2}+c^{2}$ we have

$$
\begin{aligned}
J^{\prime}(x, \theta) \ll & \int_{x}^{2 x}\left\{\theta^{2}\left(\mathfrak{M}(t)-\frac{t}{\log t}\right)^{2}+\mathfrak{R}(t, \theta)^{2}\right\} d t \\
& +\theta^{2} x^{3} \mathcal{L}^{3 k-2}\left(\delta^{-\delta / 3}+\mathcal{L}^{3 k} \delta^{-2 \delta / 3}\right) .
\end{aligned}
$$


The error term is $\ll_{A} x^{3} \theta^{2} \mathcal{L}^{-A}$ for any fixed $A$, provided that $\delta \geq \log \mathcal{L}$, which we assume. Hence by Lemma 1 and (2.13) we have proved

LEMma 5. The Theorem follows from the estimates

$$
\begin{gathered}
\int_{x}^{2 x}\left(\mathfrak{M}(t)-\frac{t}{\log t}\right)^{2} d t \ll \frac{x^{3}}{\mathcal{L}^{2}}\left(\varepsilon(x)+\frac{\log \log x}{\log x}\right)^{2}, \\
\int_{x}^{2 x}|\mathfrak{R}(t, \theta)|^{2} d t \ll \frac{x^{3} \theta^{2}}{\mathcal{L}^{2}}\left(\varepsilon(x)+\frac{\log \log x}{\log x}\right)^{2}
\end{gathered}
$$

uniformly for $x^{-5 / 6-\varepsilon(x)} \leq \theta \leq 1$, provided that $\delta \geq \max \left(\log \mathcal{L},\left(\log \log z_{1}\right)^{2}\right)$.

We shall prove the first part of Lemma 5 in Section 5 by taking $\theta$ "large", whereas the proof of the other estimate is achieved by means of mean-value bounds as described below.

3. The case $k \leq 2$ : reduction to mean-value estimates. For brevity we write $s=s(\tau)=1 / 2+i \tau$ throughout this section. By Perron's formula (see Lemma 3.12 of [10]) we have

$$
\begin{aligned}
\Sigma(h, t, \theta)= & \frac{1}{2 \pi i} \sum_{m=1}^{N(h)} c_{m, h} \int_{-T_{0}}^{T_{0}} W(s ; m, h) \frac{t^{s}-(t-\theta t)^{s}}{s} d \tau \\
& +O\left(\sum_{j=0}^{1} \sum_{m=1}^{N(h)}\left|c_{m, h}\right| \sum_{n=N_{1}(m)+1}^{N_{2}(m)}\left|e_{m, h}(n)\right|\left(\frac{x}{n}\right)^{1 / 2}\right. \\
& \left.\times \min \left(1, T_{0}^{-1}\left|\log \frac{t-j \theta t}{n}\right|^{-1}\right)\right) .
\end{aligned}
$$

The error term is estimated in Section 6 where we prove that

$$
\begin{aligned}
\Sigma(h, t, \theta)= & \frac{1}{2 \pi i} \sum_{m=1}^{N(h)} c_{m, h} \int_{-T_{0}}^{T_{0}} W(s ; m, h) \frac{t^{s}-(t-\theta t)^{s}}{s} d \tau \\
& +O\left(\frac{x}{T_{0}} e^{2 I}\left(\log N_{7}\right)^{3 h}\right)
\end{aligned}
$$

where

$$
N_{7}:=\max _{1 \leq m \leq N(h)} N_{2}(m) .
$$

The main term of $\Sigma$ will come from a short interval: for $|\tau| \leq T_{1}$ we have

$$
\frac{t^{s}-(t-\theta t)^{s}}{s}=\theta t^{s}+O\left(|s| \theta^{2} t^{1 / 2}\right) .
$$


Hence, setting $S_{0}=S_{0}(h):=\sum_{m=1}^{N(h)}\left|c_{m, h}\right|$,

$$
\begin{gathered}
\mathfrak{M}(h, t):=\frac{1}{2 \pi i} \sum_{m=1}^{N(h)} c_{m, h} \int_{-T_{1}}^{T_{1}} W(s(\tau) ; m, h) t^{s} d \tau, \\
J_{0}=J_{0}(h):=\max _{1 \leq m \leq N(h)} \int_{-T_{1}}^{T_{1}}|W(s(\tau) ; m, h)| d \tau,
\end{gathered}
$$

we have

$$
\begin{aligned}
\frac{1}{2 \pi i} \sum_{m=1}^{N(h)} c_{m, h} \int_{-T_{1}}^{T_{1}} W(s ; m, h) \frac{t^{s}-(t-\theta t)^{s}}{s} d \tau & \\
& =\theta \mathfrak{M}(h, t)+O\left(T_{1} J_{0} S_{0} \theta^{2} x^{1 / 2}\right) .
\end{aligned}
$$

Summing up, from (3.1)-(3.5) we have

$$
\begin{aligned}
\Sigma(h, t, \theta)= & \theta \mathfrak{M}(h, t)+\mathfrak{R}_{1}(h, t, \theta) \\
& +\frac{1}{2 \pi i} \sum_{m=1}^{N(h)} c_{m, h}\left\{\int_{-T_{0}}^{-T_{1}}+\int_{T_{1}}^{T_{0}}\right\} W(s ; m, h) \frac{t^{s}-(t-\theta t)^{s}}{s} d \tau \\
= & \theta \mathfrak{M}(h, t)+\mathfrak{R}_{1}(h, t, \theta)+\mathfrak{R}_{2}(h, t, \theta)
\end{aligned}
$$

say, where $\mathfrak{M}(h, t)$ is independent of $\theta$. The ranges $\left[-T_{0},-T_{1}\right]$ and $\left[T_{1}, T_{0}\right]$ are dealt with by means of the following mean-value bound, which will be proved in Section 7.

Lemma 6. There is a constant $C_{0}>0$ with the following property. Let

$$
\eta=\eta(T):=C_{0}(\log T)^{-2 / 3}(\log \log T)^{-1 / 3}
$$

and

$$
\mathcal{E}:=\exp \left\{\left(\frac{\mathcal{L}}{\log z_{1}}\right)^{2} \log \log z_{1}\right\}
$$

and assume that $z_{1}=z_{1}(x)$ and $\delta=\delta(x)$ are functions of $x$ such that $\delta \geq\left(\log \log z_{1}\right)^{2}, \log z_{1} \geq \mathcal{L}^{2 / 3}, z_{2}=z_{1}^{\delta}=x^{o(1)}$ and $\mathcal{E}=x^{o(1)}$. Then for each fixed $\alpha \in(0,1 / 12)$ there exists $\beta=\beta(\alpha)$ with $\beta \in(0,1 / 42)$ with the following property. Let

$$
x^{1 / 4}<z \leq x^{1 / 3-\alpha} \quad \text { and } \quad 3 \leq T \leq T_{0}=x^{5 / 6+\beta} .
$$

Then for $t \in[x, 2 x]$ and $h \leq 2$ we have

$$
\int_{T}^{2 T}|W(s(\tau) ; m, h)|^{2} d \tau \ll x \mathcal{E}^{2 h^{2}}\left(z_{1}^{-\eta / 6}+T^{-1 / 6}\right) .
$$


We obviously have

$$
\mathfrak{R}_{2}(h, t, \theta) \ll \sum_{m=1}^{N(h)}\left|c_{m, h}\right|\left|\int_{T_{1}}^{T_{0}} W(s ; m, h) \frac{t^{s}-(t-\theta t)^{s}}{s} d \tau\right|
$$

and this means that

$$
\begin{aligned}
& \int_{x}^{2 x}\left|\Re_{2}(h, t, \theta)\right|^{2} d t \\
& \qquad S_{0}^{2} \max _{1 \leq m \leq N(h)} \int_{x}^{2 x}\left|\int_{T_{1}}^{T_{0}} W(s ; m, h) \frac{t^{s}-(t-\theta t)^{s}}{s} d \tau\right|^{2} d t .
\end{aligned}
$$

The next lemma is needed to invert the order of integration.

Lemma 7. Let $F(s)$ be a continuous complex-valued function. Then for $1 \leq T_{1} \leq T_{0} \leq x$ and $s=1 / 2+i \tau$ we have

$$
\int_{x}^{2 x}\left|\int_{T_{1}}^{T_{0}} F(s) \frac{t^{s}-(t-\theta t)^{s}}{s} d \tau\right|^{2} d t \ll x^{2} \theta^{2} \mathcal{L}^{2} \max _{T_{1} \leq T \leq T_{0}} \int_{T}^{2 T}|F(s)|^{2} d \tau .
$$

A proof can be easily given by squaring out the integral, performing the integration with respect to $t$ first and then using the elementary inequality $|a b| \leq|a|^{2}+|b|^{2}$ on the remaining double integral. A form of this result appears as Lemma 2 in Harman [2] and elsewhere. We omit the details for brevity.

We remark that $\mathcal{L}^{A} \ll_{A} \mathcal{E}$ for any fixed $A$, that $N_{7} \ll 2^{2 h+I} x \ll \mathcal{E} x$ and that the definition of $W$ easily implies $J_{0} \ll T_{1} x^{1 / 2}$. The next lemma is proved in Section 6.

Lemma 8. For large enough $x$ we have

$$
\left|S_{0}\right| \ll \exp \left\{h \frac{\mathcal{L}}{\log z_{1}}(\log \mathcal{L})^{2}\right\} .
$$

Hence $\mathcal{L}^{2} S_{0}^{2} \ll \mathcal{E}$. We now choose $k_{0}:=4$ and set

$$
\begin{aligned}
& \mathfrak{M}_{1}(t):=\sum_{k=1}^{2} \sum_{h=0}^{k} \alpha(h, k) \mathfrak{M}(h, t), \\
& \mathfrak{R}_{j}(t, \theta):=\sum_{k=1}^{2} \sum_{h=0}^{k} \alpha(h, k) \mathfrak{R}_{j}(h, t, \theta),
\end{aligned}
$$

for $j=1,2$. Summing up, from Lemmas 4, 6-8, and from (3.2), (3.5)-(3.8) we have

(3.9) $\pi(t)-\pi(t-\theta t)-\frac{1}{3} \sum_{n \in \mathcal{I}(t, \theta)} a_{3}(n)=\theta \mathfrak{M}_{1}(t)+\mathfrak{R}_{1}(t, \theta)+\mathfrak{R}_{2}(t, \theta)$, 
where

$$
\begin{gathered}
\mathfrak{R}_{1}(t, \theta) \ll x \mathcal{E} T_{0}^{-1}+x \theta^{2} \mathcal{E} T_{1}^{2}, \\
\int_{x}^{2 x}\left|\Re_{2}(t, \theta)\right|^{2} d t \ll x^{3} \theta^{2} \mathcal{E}^{9}\left(z_{1}^{-\xi / 6}+T_{1}^{-1 / 6}\right),
\end{gathered}
$$

and $\xi:=\eta\left(T_{1}\right)$. We finally choose our parameters as follows. First we choose $\delta:=(\log \mathcal{L})^{2}$ so that $\delta \geq \max \left(\log \mathcal{L},\left(\log \log z_{1}\right)^{2}\right)$ if $z_{1} \leq x$, and $z_{2}=x^{o(1)}$ provided that $\log z_{1}=o\left(\mathcal{L}(\log \mathcal{L})^{-2}\right)$. Next, we choose $T_{1}:=\mathcal{E}^{55}$ and observe that $T_{1}$ tends to infinity with $x$. The choice

$$
z_{1}:=\exp \left\{\mathcal{L}^{8 / 9} \log \mathcal{L}\right\}
$$

implies

$$
z_{1}^{-\xi} \ll_{A} \mathcal{E}^{-A},
$$

for any fixed $A$. We now see that the hypotheses of Lemma 6 are satisfied and (3.9)-(3.11) finally yield

Lemma 9. Let $\alpha, \beta$ and $z$ be as in Lemma 6. For $t \in[x, 2 x]$ there exist functions $\mathfrak{M}_{1}(t)$ and $\mathfrak{R}^{\prime}(t, \theta)$ such that

$$
\pi(t)-\pi(t-\theta t)-\frac{1}{3} \sum_{n \in \mathcal{I}(t, \theta)} a_{3}(n)=\theta \mathfrak{M}_{1}(t)+\mathfrak{R}^{\prime}(t, \theta),
$$

where $\mathfrak{M}_{1}(t)$ is independent of $\theta$ and

$$
\int_{x}^{2 x}\left|\Re^{\prime}(t, \theta)\right|^{2} d t \ll_{A} x^{3} \theta^{2} \mathcal{L}^{-A},
$$

for any fixed $A$, provided that

$$
x^{-5 / 6-\beta} \leq \theta \leq \exp \left\{-100 \mathcal{L}^{2 / 9}\right\} .
$$

4. The case $k=3$ : reduction to mean-value estimates. The analysis of the case $k=3$ is quite similar to the previous one, but we have to be slightly more careful in order to obtain a good error term. We exploit the fact that each Dirichlet polynomial we use is the product of only 3 factors, as opposed to Section 3 where the number of factors was $2 h+I$. Define

$$
P(s):=\sum_{z \leq p \leq 2 x} \frac{1}{p^{s}} \text { and } P^{*}(s):=\sum_{z_{3} \leq p \leq 2 x} \frac{1}{p^{s}},
$$

where $z_{3}$ is a new parameter satisfying $z \leq z_{3} \leq x^{1 / 3}$. Note that if $n \leq 2 x$ then $a_{3}(n)$ is precisely the coefficient of $n^{-s}$ in $P(s)^{3}$. Let $b_{3}(n)$ be the coefficient of $n^{-s}$ in $P^{*}(s)^{3}$. We write $P_{1}(s)=P(s)-P^{*}(s)$ so that $a_{3}(n)-$ 
$b_{3}(n)$ is the coefficient of $n^{-s}$ in

$$
P(s)^{3}-P^{*}(s)^{3}=\sum_{j=1}^{3}\left(\begin{array}{l}
3 \\
j
\end{array}\right) P_{1}(s)^{j} P^{*}(s)^{3-j}
$$

if $n \leq t$. We write

$$
P_{1}(s)=\sum_{-E \leq e \leq 0} P_{e}(s) \text { and } P^{*}(s)=\sum_{1 \leq e \leq F} P_{e}(s),
$$

where $E$ and $F$ are integers satisfying $2^{-E-1} z_{3} \leq z<2^{-E} z_{3}$ and $2^{F-1} z_{3} \leq$ $2 x<2^{F} z_{3}$, and

$$
P_{e}(s):=\sum_{\substack{2^{e-1} \\ z \leq p \leq p<2^{e}}} \frac{1}{p^{s}} .
$$

Since $E, F \ll \mathcal{L}$, for some $M \ll \mathcal{L}^{3}$ and $c_{m} \ll 1$ we have

$$
P(s)^{3}-P^{*}(s)^{3}=\sum_{m=1}^{M} c_{m} P(s ; m) \quad \text { where } \quad P(s ; m):=\prod_{j=1}^{3} P_{e_{j}}(s)
$$

with $e_{1} \leq 0$. Write $V_{j}:=2^{e_{j}-1} z_{3}$ so that

$$
P(s ; m)=\sum_{N_{8} \leq n \leq N_{9}} \frac{f_{m}(n)}{n^{s}},
$$

say, where $N_{8}:=\prod_{j} V_{j}$ and $N_{9}:=2^{3} N_{8}$. As above, we discard those $P(s ; m)$ having either $N_{8} \geq t$ or $N_{9} \leq t / 2$ and relabel the remaining ones so that for some $N \leq M$ we have

$$
\sum_{n \in \mathcal{I}(t, \theta)} a_{3}(n)=\sum_{n \in \mathcal{I}(t, \theta)} b_{3}(n)+\sum_{m=1}^{N} \sum_{n \in \mathcal{I}(t, \theta)} f_{m}(n) .
$$

The same analysis of Section 3 , with the bound $\left|f_{m}(n)\right| \leq 3$ !, yields

$$
\sum_{n \in \mathcal{I}(t, \theta)} f_{m}(n)=\frac{1}{2 \pi i} \int_{1 / 2-i T_{2}}^{1 / 2+i T_{2}} P(s ; m) \frac{t^{s}-(t-\theta t)^{s}}{s} d s+O\left(\frac{x \mathcal{L}}{T_{2}}\right),
$$

for $T_{2} \leq x$. The ranges $\left[-T_{2},-T_{3}\right]$ and $\left[T_{3}, T_{2}\right]$ are treated by means of the following mean-value bound, which will be proved in Section 8 .

Lemma 10. Let $x^{19 / 60} \leq z \leq x^{1 / 3}$ and $x^{5 / 6} \leq T_{2} \leq x^{11 / 12}$. Then, if $P(s ; m)$ is as above with $V_{3} \geq V_{2} \geq V_{1} \geq z / 2$, we have

$$
\int_{T}^{2 T}\left|P\left(\frac{1}{2}+i \tau ; m\right)\right|^{2} d \tau \ll x \mathcal{L}^{62}\left(z_{1}^{-\eta / 6}+T^{-1 / 6}+\left(T_{2} V_{3}^{-5 / 2}\right)^{1 / 9}\right)
$$

uniformly for $3 \leq T \leq T_{2}$, where $\eta$ is given by (3.7). 
We proceed precisely as in Section 3, using Lemma 7 again with $F(s)=$ $P(s ; m)$ and $(3.3)$ for the range $\left[-T_{3}, T_{3}\right]$, obtaining

$$
\sum_{n \in \mathcal{I}(t, \theta)} f_{m}(n)=\theta \frac{1}{2 \pi i} \int_{1 / 2-i T_{3}}^{1 / 2+i T_{3}} P(s ; m) t^{s} d s+\mathfrak{R}_{1}(3, t, \theta)+\mathfrak{R}_{2}(3, t, \theta),
$$

where

$$
\begin{gathered}
\mathfrak{R}_{1}(3, t, \theta) \ll x \mathcal{L} T_{2}^{-1}+x \theta^{2} T_{3}^{2}, \\
\int_{x}^{2 x}\left|\Re_{2}(3, t, \theta)\right|^{2} d t \ll x^{3} \theta^{2}\left(z_{1}^{-\varrho / 3}+T_{3}^{-1 / 3}+\left(T_{2} V_{3}^{-5 / 2}\right)^{1 / 9}\right) \mathcal{L}^{62},
\end{gathered}
$$

and $\varrho=\eta\left(T_{2}\right)$. Since $V_{3}^{2} \geq x z_{3}^{-1}$ we have $T_{2} V_{3}^{-5 / 2} \ll T_{2} z_{3}^{5 / 4} x^{-5 / 4}$. We finally choose the parameters: Let $\nu$ be a sufficiently large positive constant and set $T_{2}:=\mathcal{L}^{\nu} \max \left(\theta^{-1}, x^{5 / 6}\right), T_{3}:=\mathcal{L}^{\nu}$ and also $x^{19 / 60} \leq z_{3} \leq$ $\mathcal{L}^{-\nu} \min \left(\theta^{4 / 5} x, x^{1 / 3}\right)$. Then (4.1)-(4.4) imply

$$
\sum_{n \in \mathcal{I}(t, \theta)} a_{3}(n)=\sum_{n \in \mathcal{I}(t, \theta)} b_{3}(n)+\theta \mathfrak{M}_{3}\left(t, z_{3}\right)+\mathfrak{R}^{\prime \prime}\left(t, \theta, z_{3}\right),
$$

say, where $\mathfrak{M}_{3}\left(t, z_{3}\right)$ is independent of $\theta$ and

$$
\int_{x}^{2 x}\left|\Re^{\prime \prime}\left(t, \theta, z_{3}\right)\right|^{2} d t \ll x^{3} \theta^{2} \mathcal{L}^{60-\nu / 18},
$$

provided that $\theta$ satisfies (3.12). Now choose $z:=x^{19 / 60}$, so that the hypotheses of both Lemmas 6 and 10 are satisfied, and take $\nu:=1500$. Hence, from Lemma 9, (4.5) and (4.6) we deduce

LEMma 11. There exists a small positive constant $\lambda$ such that if

$$
x^{-5 / 6-\lambda} \leq \theta \leq \exp \left\{-100 \mathcal{L}^{2 / 9}\right\}
$$

and

$$
x^{19 / 60} \leq w \leq \mathcal{L}^{-1500} \min \left(\theta^{4 / 5} x, x^{1 / 3}\right)
$$

then for $t \in[x, 2 x]$ there exists a function $\mathfrak{M}(t, w)$ independent of $\theta$ such that

$$
\pi(t)-\pi(t-\theta t)-\frac{1}{3} \sum_{n \in \mathcal{I}(t, \theta)} b_{3}(n)=\theta \mathfrak{M}(t, w)+\mathfrak{R}(t, \theta, w)
$$

where

$$
\int_{x}^{2 x}|\mathfrak{R}(t, \theta, w)|^{2} d t \ll x^{3} \theta^{2} \mathcal{L}^{-20} .
$$



that

It now remains to estimate the contribution of $b_{3}(n)$. First we remark

$$
\int_{x}^{2 x}\left|\sum_{n \in \mathcal{I}(t, \theta)} b_{3}(n)\right|^{2} d t \ll\left(\sup _{t \in[x, 2 x]} \sum_{n \in \mathcal{I}(t, \theta)} b_{3}(n)\right) \int_{x}^{2 x} \sum_{n \in \mathcal{I}(t, \theta)} b_{3}(n) d t,
$$

and that a simple argument based on the Brun-Titchmarsh inequality gives

$$
\begin{aligned}
\int_{x}^{2 x} \sum_{n \in \mathcal{I}(t, \theta)} b_{3}(n) d t & \ll \sum_{x-\theta x<n \leq 2 x} b_{3}(n) \int_{\max (x, n)}^{\min \left(2 x, n(1-\theta)^{-1}\right)} d t \\
& \ll \theta x \sum_{n \leq 2 x} b_{3}(n) \ll \theta x \sum_{w \leq p, q \leq 2 x / w^{2}} \sum_{r \leq 2 x /(p q)} 1 \\
& \ll \frac{\theta x^{2}}{\mathcal{L}}\left(\sum_{w \leq p \leq 2 x / w^{2}} \frac{1}{p}\right)^{2} \ll \frac{\theta x^{2}}{\mathcal{L}}\left(\frac{\log \left(x w^{-3}\right)}{\mathcal{L}}\right)^{2} .
\end{aligned}
$$

The same argument leading to (4.10) shows that the expected order of magnitude for the supremum over $t$ in $(4.9)$ is $\theta x \mathcal{L}^{-1}\left(\log \left(x w^{-3}\right) / \mathcal{L}\right)^{2}$, and this would imply the Theorem with the exponent 2 attached to the last factor replaced by 4 . But we are unable to prove such a good bound. By Theorem 3.4 of Halberstam-Richert [1] we find

$$
\sup _{t \in[x, 2 x]} \sum_{n \in \mathcal{I}(t, \theta)} b_{3}(n) \ll \frac{\theta x}{\mathcal{L}},
$$

the lower bound in (4.7) ensuring that we save a log factor over the trivial estimate. We collect these results in the form of

Lemma 12. Let $\theta$ and $w$ be as in the statement of Lemma 11. Then

$$
\int_{x}^{2 x}\left|\sum_{n \in \mathcal{I}(t, \theta)} b_{3}(n)\right|^{2} d t \ll \frac{\theta^{2} x^{3}}{\mathcal{L}^{2}}\left(\frac{\log \left(x w^{-3}\right)}{\mathcal{L}}\right)^{2} .
$$

5. Conclusion of the proof: the main term. Here we choose $\theta$ as large as possible, i.e. $\theta=\theta_{0}:=\exp \left(-100 \mathcal{L}^{2 / 9}\right)$, and any $w$ satisfying (4.7). The Prime Number Theorem gives

$$
\pi(t)-\pi\left(t-\theta_{0} t\right)=\frac{\theta_{0} t}{\log t}+O\left(\frac{x \theta_{0}^{2}}{\mathcal{L}^{2}}\right) .
$$

Hence (4.8) yields

$$
\theta_{0}\left(\mathfrak{M}(t, w)-\frac{t}{\log t}\right)=-\frac{1}{3} \sum_{n \in \mathcal{I}\left(t, \theta_{0}\right)} b_{3}(n)-\mathfrak{R}\left(t, \theta_{0}, w\right)+O\left(\frac{x \theta_{0}^{2}}{\mathcal{L}^{2}}\right)
$$


so that by Lemmas 11 and 12 we have

$$
\theta_{0}^{2} \int_{x}^{2 x}\left(\mathfrak{M}(t, w)-\frac{t}{\log t}\right)^{2} d t \ll \frac{x^{3} \theta_{0}^{2}}{\mathcal{L}^{2}}\left(\frac{\log \left(x w^{-3}\right)}{\mathcal{L}}\right)^{2}+\frac{x^{3} \theta_{0}^{2}}{\mathcal{L}^{20}}+\frac{x^{3} \theta_{0}^{4}}{\mathcal{L}^{4}}
$$

We finally take

$$
w:=\mathcal{L}^{-1500} \min \left(\theta^{4 / 5} x, x^{1 / 3}\right) .
$$

This choice of $w$ implies that the left hand side of (5.1) is

$$
\ll \frac{x^{3} \theta_{0}^{2}}{\mathcal{L}^{2}}\left(\varepsilon(x)+\frac{\log \log x}{\log x}\right)^{2}
$$

and the first estimate of Lemma 5 follows. The second part of Lemma 5 is a consequence of Lemmas 11 and 12 and our choice of $w$. The proof of the Theorem is therefore complete.

6. Proofs of (3.2) and Lemma 8. In order to prove (3.2) we first need the bound

$$
\sum_{m}\left|c_{m, h}\right| \cdot\left|e_{m, h}(n)\right| \leq d_{3 h}(n) .
$$

By (2.6) this sum is bounded by the coefficient of $n^{-s}$ occurring in

$$
\zeta(s)^{2 h} \prod_{m=1}^{L} \exp \left(\frac{h}{m} \Sigma_{m}(s)\right)
$$

which, in its turn, is bounded by the one in

$$
\zeta(s)^{2 h} \prod_{m \geq 1} \exp \left(\frac{h}{m} \Sigma_{m}(s)\right)
$$

and the latter is a partial product of $\zeta(s)^{h}$.

We recall that we chose $N_{2} \geq t / 2$ and that $N_{1}=2^{-2 h-I} N_{2}$ by (2.11). Setting

$$
N_{7}^{\prime}:=\min _{1 \leq m \leq N(h)} N_{1}(m),
$$

the error term with $j=0$ in $(3.1)$ is

$$
\ll 2^{I / 2} \sum_{N_{7}^{\prime}<n \leq N_{7}} d_{3 h}(n) \min \left(1, T_{0}^{-1}\left|\log \frac{t}{n}\right|^{-1}\right),
$$

since each $n$ counted in (3.1) is $\geq N_{1}(m) \geq N_{7}^{\prime} \gg x 2^{-I}$. For the sake of brevity, for $r \in \mathbb{N}$ let

$$
H_{r}=\left\{n \in\left(N_{7}^{\prime}, N_{7}\right]: r T_{0}^{-1} \leq|\log (t / n)|<(r+1) T_{0}^{-1}\right\} .
$$

Observe that $H_{r} \neq \emptyset$ only for $0 \leq r \leq M$, say, with $M \ll I T_{0}$. Then the 
sum in $(6.1)$ is

$$
\begin{aligned}
& \ll \sum_{n \in H_{0}} d_{3 h}(n)+\sum_{r=1}^{M} \sum_{n \in H_{r}} T_{0}^{-1} d_{3 h}(n)\left|\log \frac{t}{n}\right|^{-1} \\
& \ll \sum_{n \in H_{0}} d_{3 h}(n)+\sum_{r=1}^{M} \sum_{n \in H_{r}} T_{0}^{-1} d_{3 h}(n)\left(r T_{0}^{-1}\right)^{-1} \\
& \ll \sum_{r=0}^{M} \frac{1}{r+1} \sum_{n \in H_{r}} d_{3 h}(n) .
\end{aligned}
$$

Furthermore $t T_{0}^{-1} \exp \left(-r T_{0}^{-1}\right) \ll\left|H_{r}\right| \ll t T_{0}^{-1} \exp \left(r T_{0}^{-1}\right)$ for all $r \leq M$, and (3.2) follows using Lemma 3. The term with $j=1$ in (3.1) is dealt with in the same way.

For Lemma 8 we need the following elementary inequality which is easily proved by induction: For any integer $A \geq 2$ and real number $B \geq 3$ we have

$$
\sum_{n=0}^{A} \frac{B^{n}}{n !} \leq B^{A} .
$$

Arguing as in Section 5 of [4] we find, after a simple computation,

$$
\begin{aligned}
S_{0} & \leq(B+1)^{h}(C+1)^{h} \exp \left\{h \sum_{m=1}^{L / 2} \frac{L}{m} \log \frac{D+1}{m}+h \frac{L}{2} \log \frac{2 D}{L}\right\} \\
& \leq \exp \left\{h \frac{\mathcal{L}}{\log z_{1}}(\log \mathcal{L})^{2}\right\},
\end{aligned}
$$

for large enough $x$, since $B, C, D \ll \mathcal{L}$ and $z_{1}=x^{o(1)}$, and Lemma 8 follows.

\section{Proof of Lemma 6}

Preliminaries. The proof is quite similar to the proof of Lemma 8 in [4]. For the sake of brevity we do not duplicate the whole argument, but merely give the needed modifications. We say that a set $\mathcal{S}$ of points $\tau_{n} \in[T, 2 T]$ is well spaced if $\left|\tau_{m}-\tau_{n}\right| \geq 1$ for every $\tau_{m}, \tau_{n} \in \mathcal{S}$ with $n \neq m$. We write $s=1 / 2+i \tau$ and $s_{n}=1 / 2+i \tau_{n}$ throughout this section. We need an estimate for

$$
J_{1}(T):=\int_{T}^{2 T}|W(s)|^{2} d \tau .
$$

We first write $W$ as the product of $W_{1}, W_{2}$ and $W_{3}$, where

$$
\begin{aligned}
W_{1}(s) & :=\prod_{X_{i} \geq z_{1}} X_{b_{i}}(s) \prod_{i=1}^{I_{1}} Z_{d_{i}}^{(1)}(s), \quad W_{2}(s):=\prod_{X_{i}<z_{1}} X_{b_{i}}(s) \prod_{i=1}^{h} Y_{c_{i}}(s), \\
W_{3}(s) & :=W(s)\left(W_{1}(s) W_{2}(s)\right)^{-1} .
\end{aligned}
$$


We also set

$$
x_{1}:=\prod_{X_{i} \geq z_{1}} X_{i} \prod_{i=1}^{I_{1}} Z_{i}, \quad x_{2}:=\prod_{X_{i}<z_{1}} X_{i} \prod_{i=1}^{h} Y_{i}, \quad x_{3}:=\prod_{m=2}^{L} \prod_{i=1}^{I_{m}} Z_{i},
$$

so that $x_{1} x_{2} x_{3}=N_{1} \leq x$. We observe that $\left|Z_{d_{i}}^{(m)}(s)\right| \leq Z_{i}^{1-m / 2}$ for $m \geq 2$ and large enough $x$, whence $\left|W_{3}(s)\right| \leq 1$.

The main tool to obtain mean-value estimates such as our Lemmas 6 and 10 is a combination of Montgomery's mean-value bound (see Theorem 7.3 of [7]) and the Halász method. These are summarized in the following

Lemma 13. Let $K(s)$ be the Dirichlet polynomial

$$
K(s)=\sum_{n \leq K} \frac{k(n)}{n^{s}},
$$

where $K \geq 2$ and $|k(n)| \leq 1$ for every $n \leq K$. Assume that $\left|K\left(1 / 2+i \tau_{n}\right)\right| \geq$ $\mathcal{K}$ for a set $\mathcal{S}$ of well-spaced points $\tau_{n} \in[T, 2 T]$. Then, uniformly for $g \in \mathbb{N}$, we have

$$
|\mathcal{S}| \ll\left\{\mathcal{K}^{-2 g} K^{g}+T \min \left(\mathcal{K}^{-2 g}, \mathcal{K}^{-6 g} K^{g}\right)\right\} \exp \left\{6 g^{2} \log \log K\right\}(\log T K)^{5} .
$$

This is (8.4) and the following is Lemma 19 of [4].

Lemma 14. For every factor $K(s)$ of $W_{1}(s)$ we have

$$
K(s) \ll K^{1 / 2}\left(z_{1}^{-\eta}+T^{-1}\right) \mathcal{L}^{2},
$$

uniformly for $\tau \in[T, 2 T]$, where $\eta=\eta(T)$ is given by (3.7).

Actually, if $x_{3}$ is large enough, $x_{3} \geq z_{1}$, say, we see that Lemma 6 follows directly from Montgomery's mean-value bound. In fact, we have

$$
J_{1} \ll \sup _{\tau \in[T, 2 T]}\left|W_{3}(s)\right|^{2} \int_{T}^{2 T}\left|W_{1}(s) W_{2}(s)\right|^{2} d \tau \ll\left(T+x_{1} x_{2}\right) \sum_{n \leq x_{1} x_{2}} \frac{\left|c_{n}\right|^{2}}{n},
$$

for suitable coefficients $c_{n}$. The same argument leading to Lemma 13 above implies that the last sum is $\ll \mathcal{E}^{2 h^{2}}$, and the hypothesis on $x_{3}$ ensures that $T+x_{1} x_{2} \ll x z_{1}^{-1}$, which is more than enough for Lemma 6 . Hence we may assume in what follows that $x_{3} \leq z_{1}$. We remark that from the definitions above and (2.11) we have $x_{2}=x^{o(1)}$ and $x_{1}=x^{1+o(1)}$. We do not rule out the possibility that $W_{1}$ consists of a single factor $X_{b_{i}}$. We use Lemma 14 in conjunction with Montgomery's mean-value theorem if $W_{1}$ has at least one factor $X_{b_{i}}(s)$ or $Z_{d_{i}}^{(1)}(s)$ with $X_{i} \leq x^{1 / 6-\alpha}$ or $Z_{i} \leq x^{1 / 6-\alpha}$, respectively. In fact, setting $K(s)=X_{b_{i}}(s), K=X_{i}\left(\operatorname{resp} . K(s)=Z_{d_{i}}^{(1)}(s), K=Z_{i}\right)$, 
$W_{1}(s)=K(s) W_{4}(s), x_{4}=x_{1} / K$, in this case we have

$$
\begin{aligned}
J_{1} & \ll \sup _{\tau \in[T, 2 T]}\left|W_{2}(s) W_{3}(s)\right|^{2} \int_{T}^{2 T}\left|W_{1}(s)\right|^{2} d \tau \\
& \ll x_{2} K\left(z_{1}^{-2 \eta}+T^{-2}\right) \int_{T}^{2 T}\left|W_{4}(s)\right|^{2} d \tau,
\end{aligned}
$$

and the last integral is estimated by means of Montgomery's theorem, giving

$$
J_{1} \ll x_{2} K\left(z_{1}^{-2 \eta}+T^{-2}\right)\left(T+x_{4}\right) \sum_{n \leq x_{4}} \frac{\left|c_{n}^{\prime}\right|^{2}}{n},
$$

for suitable coefficients $c_{n}^{\prime}$. As above, the last sum is $\ll \mathcal{E}^{2 h^{2}}$, and the hypothesis on $K$ ensures that Lemma 6 follows in this case, with $\beta=\alpha / 2$.

From now on we may assume that every factor $K(s)$ of $W_{1}(s)$ has $K \geq$ $x^{1 / 6-\alpha}$. Thus we have $I_{1} \leq 12$ and there exists a set $\mathcal{S}$ of $\ll T$ well-spaced points $\tau_{n} \in[T, 2 T]$ such that

$$
J_{1} \ll \sum_{\tau_{n} \in \mathcal{S}}\left|W\left(s_{n}\right)\right|^{2} .
$$

The contribution to the sum of the points $\tau_{n}$ for which some factor of $W_{1}$ is $\leq x^{-1}$ is easily seen to be $\ll T$. We discard these points, and from now on assume that each factor of $W_{1}$ is $\geq x^{-1}$. Then we split the range for each factor of $W_{1}(s)$ into dyadic intervals $\left[D_{j}, 2 D_{j}\right.$ ) (if the factor is an $X_{b_{i}}(s)$ ) or $\left[E_{j}, 2 E_{j}\right.$ ) (if the factor is a $Z_{d_{i}}^{(1)}(s)$ ), where

$$
x^{-1} \ll D_{j}=2^{d} \ll X_{i}^{1 / 2} \text { and } x^{-1} \ll E_{j}=2^{e} \ll Z_{i}^{1 / 2}
$$

for some integers $d$ and $e$. We observe that our hypothesis that each factor of $W_{1}(s)$ is not too small ensures that the number of ranges (that is, the number of values taken by $d$ and $e$ above) is $\leq C_{2} \mathcal{L}$ in each case, for some absolute constant $C_{2}$. For brevity we write $\mathcal{L}_{0}=2 C_{2} \mathcal{L}$. We may divide the remaining points into at most $\left(\mathcal{L}_{0} / 2\right)^{h+I_{1}}$ classes $\mathcal{S}(\mathbf{D}, \mathbf{E})$ where $\mathbf{D}=\left(D_{1}, \ldots, D_{h}\right)$ and $\mathbf{E}=\left(E_{1}, \ldots, E_{I_{1}}\right)$, for which

$$
\left|X_{b_{i}}\left(s_{n}\right)\right| \in\left[D_{i}, 2 D_{i}\right) \quad \text { and } \quad\left|Z_{d_{i}}^{(1)}\left(s_{n}\right)\right| \in\left[E_{i}, 2 E_{i}\right)
$$

We write

$$
\mathcal{P}(\mathbf{D}, \mathbf{E}):=\prod_{i} D_{i} \prod_{i} E_{i}
$$

As above, we estimate $W_{2}(s)$ trivially and conclude that

Lemma 15. There exists a set $\mathcal{S}(\mathbf{D}, \mathbf{E})$ of well-spaced points $\tau_{n} \in[T, 2 T]$ satisfying (7.1) and such that

$$
J_{1} \ll T+x_{2} \mathcal{P}(\mathbf{D}, \mathbf{E})^{2}|\mathcal{S}(\mathbf{D}, \mathbf{E})| \mathcal{L}_{0}^{h+I_{1}} .
$$


We shall give upper bounds for $|\mathcal{S}|$ by means of Lemmas 13 and 14 . Since these bounds are essentially the same as in [4] we simply quote the results.

LEMMA 16. If the hypotheses of Lemma 13 hold for $K(s)=X_{i}(s)$ with $K=2 X_{i} \geq T^{1 / 2}$ then either

$$
\mathcal{K} \ll K^{1 / 2} T^{-1}(\log K)^{3}
$$

or

$$
|\mathcal{S}| \ll \mathcal{K}^{-4} T(\log K)^{9} .
$$

This is Lemma 18 of [4].

If (7.2) holds, the trivial bound $|\mathcal{S}| \ll T$ and Lemmas 15 and 16 imply

Lemma 17. If $X_{i} \geq \frac{1}{2} T^{1 / 2}$ for some $i$ then either

$$
|\mathcal{S}| \ll \mathcal{K}^{-4} T(\log K)^{9}
$$

or

$$
J_{1} \ll T+x_{1} x_{2} T^{-1} \mathcal{L}_{0}^{3+h+I_{1}} .
$$

The second estimate is proved taking $\mathcal{K}=D_{i}$ in (7.2) and observing that the definition implies that $\mathcal{P} \ll\left|W_{1}\left(s_{n}\right)\right|$. Since $\mathcal{L}_{0}^{3+h+I_{1}} \ll \mathcal{E}$ and $x_{1} x_{2} \leq x$, (7.4) yields the conclusion of Lemma 6 and more.

Large factors of $W_{1}(s)$. The argument here is essentially the same as in Section 8 of [4], and Lemma 6 follows precisely in the same way, since the results in that section are bounds for $|\mathcal{S}|$. We take a factor of $W_{1}(s)$, $K(s)=X_{b_{i}}(s)$ or $Z_{d_{i}}^{(1)}(s)$, and let $K=2 X_{i}$ or $2 Z_{i}, \mathcal{K}=D_{i}$ or $E_{i}$ accordingly. We define $\sigma$ by means of $\mathcal{K}=K^{\sigma-1 / 2}$. The argument in Section 8 of [4] is as follows: if $\varphi$ is the maximum value of a $\sigma$ occurring above then

$$
\mathcal{P}(\mathbf{D}, \mathbf{E})^{2} \leq \prod_{i} D_{i}^{2 \varphi-1} \prod_{i} E_{i}^{2 \varphi-1} \leq x_{1}^{2 \varphi-1},
$$

and by Lemma 15 we have

$$
J_{1} \ll T+x x_{1}^{2 \varphi-2} \mathcal{L}_{0}^{h+I_{1}}|\mathcal{S}(\mathbf{D}, \mathbf{E})| .
$$

If $\varphi \geq 5 / 6$ then suitable choices of $g$ in Lemma 13 yield

$$
|\mathcal{S}(\mathbf{D}, \mathbf{E})| \ll\left(T^{2-2 \varphi}+z^{4-4 \varphi}\right) \mathcal{L}^{29} \mathcal{E}^{3 / 2},
$$

and the upper bounds for $T$ and $z$ in the hypothesis of Lemma 6 together with (7.5) and (7.6) yield

$$
J_{1} \ll T+x x_{1}^{(\varphi-1) / 6} \mathcal{L}_{0}^{29+h+I_{1}} \mathcal{E}^{3 / 2} .
$$

The upper bound for $x_{1}^{\varphi-1}$ which we need is provided by Lemma 14 and the inequality $K \ll x$. In conclusion, since $\mathcal{L}_{0}^{A} \ll_{A} \mathcal{E}$, we see that Lemma 6 follows if $\varphi \geq 5 / 6$. 
Conclusion of the proof of Lemma 6. In the remaining case, HeathBrown's argument leads to the stronger inequality

$$
J_{1} \ll x^{1-\gamma}
$$

for some $\gamma>0$. This follows from several bounds for $|\mathcal{S}|$ which are essentially the same as in our case. We very briefly sketch the argument, without entering into the details. First the hypotheses of Lemma 6 ensure that

$$
J_{1} \ll T+x^{o(1)} \mathcal{P}^{2}|\mathcal{S}| \text {. }
$$

By means of Lemma 13 we prove the following bounds: If $K(s)=X_{b_{i}}(s)$ then

$$
|\mathcal{S}| \ll \begin{cases}T^{12(1-\sigma) / 5} x^{o(1)} & \text { in any case, } \\ \left(T / X_{i}\right)^{4-4 \sigma} x^{o(1)} & \text { if } T^{2 / 5} \leq X_{i} \leq T^{1 / 2}, \\ T^{2-2 \sigma} x^{o(1)} & \text { if } X_{i} \geq T^{1 / 2}\end{cases}
$$

and if $K(s)=Z_{d_{i}}^{(1)}(s)$ then

$$
|\mathcal{S}| \ll T^{12(1-\sigma) / 5} x^{o(1)} .
$$

Using these bounds we see that (7.7) holds provided that the following conditions hold.

First case. If $X_{i} \geq x^{1 / 3+\delta}$ for some $\delta \geq \beta$ and $\sigma \geq \varphi-\varepsilon$ we need to have

$$
\gamma<\min \left(\frac{1}{6}-\beta, \frac{1}{18}-\frac{1}{3} \beta-2 \varepsilon, \frac{2}{3} \delta-\frac{2}{3} \beta-2 \varepsilon\right) \text {. }
$$

Second case. If $X_{i} \geq x^{1 / 3+\delta}$ for some $\delta \geq \beta$ and $\sigma \leq \varphi-\varepsilon$ we need to have

$$
\gamma<\min \left(\frac{1}{6}-\beta, \frac{2}{3} \varepsilon-\beta\right) .
$$

Third case. If $X_{i} \leq x^{1 / 3+\delta}$ for all $i$ we need

$$
\gamma<\min \left(\frac{1}{6}-\beta, \frac{2}{3} \varepsilon-\beta-4 \delta \varepsilon, \frac{1}{6} \alpha-\frac{1}{3} \beta-2 \varepsilon\right) .
$$

Now, we easily see that the choices

$$
\delta=\frac{1}{30}, \quad \beta=\frac{1}{30} \alpha, \quad \varepsilon=\frac{1}{15} \alpha
$$

allow the choice $\gamma=\alpha / 50$ and satisfy the hypotheses of Lemma 6 .

8. Proof of Lemma 10. This lemma is proved in a similar fashion to Lemma 11 in [4] and we simply sketch the argument, with the necessary changes. As in Section 10 of [4], let $\mathbf{F}=\left(F_{1}, F_{2}, F_{3}\right)$ and $\mathcal{S}(\mathbf{F})$ be a set of well-spaced points $\tau_{n} \in[T, 2 T]$ such that

$$
F_{i} \leq\left|P_{e_{i}}\left(1 / 2+i \tau_{n}\right)\right|<2 F_{i} \quad \text { for } i=1,2,3 .
$$

The same argument of Section 7 gives

$$
\int_{T}^{2 T}|P(1 / 2+i \tau)|^{2} d \tau \ll T_{2}+\mathcal{L}^{3}|\mathcal{S}(\mathbf{F})| \prod_{i=1}^{3} F_{i}^{2}
$$


for some F. Fix an index $i$ and set $\mathcal{K}=F_{i}=V_{i}^{\sigma-1 / 2}$ and $K=2 V_{i}$. We remark that our choice of parameters implies that

$$
T_{2}^{1 / 3} \ll K \ll T_{2}^{1 / 2} .
$$

We use Lemma 13 with several different values of $g$. First, if $\varphi=\max \sigma \geq$ $5 / 6$, we choose $g=2$ and (8.2) implies that

$$
|\mathcal{S}(\mathbf{F})| \ll T_{2}^{2-2 \varphi} \mathcal{L}^{29}
$$

and Lemma 10 easily follows as in [4], on substituting into (8.1), since $\prod F_{i}^{2} \leq \prod V_{i}^{2 \varphi-1} \leq x^{2 \varphi-1}$. An upper bound for $x^{\varphi-1}$ is provided by Lemma 14 . In the other case, choose $g=3$ to obtain

$$
|\mathcal{S}(\mathbf{F})| \ll K^{6-6 \sigma} \mathcal{L}^{59}
$$

or $g$ in such a way that $T_{2} K^{-1 / 2} \leq K^{g} \leq T_{2} K^{1 / 2}$. In the latter case we have

$$
|\mathcal{S}(\mathbf{F})| \ll\left(T K^{1 / 2}\right)^{2-2 \sigma} \mathcal{L}^{59}
$$

since $g \leq 3$ anyway. Since now $\sigma \leq 5 / 6,(8.3)$ and (8.4) imply

$$
|\mathcal{S}(\mathbf{F})| \ll K^{6-6 \sigma}\left(T_{2} K^{-5 / 2}\right)^{1 / 3} \mathcal{L}^{59}
$$

when $K \leq T_{2}^{2 / 5}$ and when $K \geq T_{2}^{2 / 5}$ respectively. This means that

$$
\begin{aligned}
& F_{i}^{6}|\mathcal{S}(\mathbf{F})| \ll\left(K^{\sigma-1 / 2}\right)^{6} K^{6-6 \sigma} \mathcal{L}^{59}=K^{3} \mathcal{L}^{59}, \\
& F_{i}^{6}|\mathcal{S}(\mathbf{F})| \ll\left(K^{\sigma-1 / 2}\right)^{6} K^{6-6 \sigma}\left(T_{2} K^{-5 / 2}\right)^{1 / 3} \mathcal{L}^{59}=K^{3}\left(T_{2} K^{-5 / 2}\right)^{1 / 3} \mathcal{L}^{59} .
\end{aligned}
$$

We use the former for $i=1,2$, and the latter for $i=3$, take their geometric mean, and from (8.1) we obtain Lemma 10 in this case too, since $F_{i}^{2} \leq$ $V_{i}^{2 \sigma-1} \leq V_{i}$.

9. Some comments. The knowledgeable reader sees at once that we had to make a different choice for the Dirichlet polynomials from Heath-Brown [4]. Indeed, the choice therein leads to too large error terms in Lemma 4 since we have a larger $z$ than Heath-Brown and a much smaller $h$. This is due to the fact that we need $z$ to be almost $x^{1 / 3}$, since we have the same problems he encounters in Section 9 when the product $W$ has 6 factors, but already with only 3 factors. The slight additional difficulty is more than compensated by the fact that we only have to save a little over the estimate given by Montgomery's theorem, since our problem leads naturally to estimating the mean-square of a Dirichlet polynomial.

We did not use Watt's mean-value bound (Theorem 2 of [12]) in proving Lemma 6 , because the hypothesis $T \geq K^{4}$ (in our notation) limits the former's usefulness in this problem to a subrange of the values of the parameters in Lemma 6 . In particular, the case when some function $X_{b_{i}}(s)$ or $Z_{d_{i}}(s)$ has length $K\left(=X_{i}\right.$ or $Z_{i}$ resp. $)$ bounded by $x^{1 / 6-\alpha}$ can be more 
easily handled by means of Montgomery's theorem alone. Compare the comment following the proof of Proposition 2.2 in [12] with the hypothesis of our Lemma 17. Even the more general Theorem 1 of Watt's paper [11] has, essentially, the same disadvantage.

\section{References}

[1] H. Halberstam and H. E. Richert, Sieve Methods, Academic Press, London, 1974 .

[2] G. Harman, Primes in short intervals, Math. Z. 180 (1982), 335-348.

[3] D. R. Heath-Brown, Prime numbers in short intervals and a generalized Vaughan identity, Canad. J. Math. 34 (1982), 1365-1377.

[4] - , The number of primes in a short interval, J. Reine Angew. Math. 389 (1988), $22-63$.

[5] M. N. Huxley, On the difference between consecutive primes, Invent. Math. 15 (1972), 164-170.

[6] C. Jia, Almost all short intervals containing prime numbers, Acta Arith. 76 (1996), 21-84.

[7] H. L. Montgomery, Topics in Multiplicative Number Theory, Lecture Notes in Math. 227, Springer, Berlin, 1971.

[8] B. Saffari and R. C. Vaughan, On the fractional parts of $x / n$ and related sequences. II, Ann. Inst. Fourier (Grenoble) 27 (2) (1977), 1-30.

[9] P. Shiu, A Brun-Titchmarsh theorem for multiplicative functions, J. Reine Angew. Math. 313 (1980), 161-170.

[10] E. C. Titchmarsh, The Theory of the Riemann Zeta-Function, 2nd ed., Oxford Univ. Press, 1986.

[11] N. Watt, Kloosterman sums and a mean value for Dirichlet polynomials, J. Number Theory 53 (1995), 179-210.

[12] - Short intervals almost all containing primes, Acta Arith. 72 (1995), 131-167.

[13] A. Zaccagnini, On the Selberg integral via Heath-Brown's identity, Riv. Mat. Univ. Parma 5 (1996), 205-212.

Dipartimento di Matematica

Università di Parma

via Massimo d'Azeglio 85/a

43100 Parma, Italy

E-mail: zaccagnini@prmat.math.unipr.it

Received on 26.3.1997

and in revised form on 24.6.1997 